\title{
Research on the Ubiquitous Universal Library Service Model of "Internet +" Environment
}

\author{
Chunhong Zhang, Tao Liu and Yuying Liu \\ Shenyang Aerospace University
}

\begin{abstract}
With the proposition of the "Internet +" concept, "Internet +" has already become a big trend while both the central government and local sectors are promoting the rapid development of the Internet industry. How can the library grasp the opportunity and use the network environment to transform the service of the library? The key is to explore the service model of "Internet + Library" and create a new type of library in the "Internet + " environment. This mode can satisfy users' demands at any time without being restricted by time and space. This paper elaborates on the concept of "Internet+", discusses the changes brought by "Internet + " to traditional libraries, and the proposes the features and service modes of ubiquitous libraries.
\end{abstract}

Keywords: Internet+; Ubiquitous; New library; Service mode

\section{“互联网+”环境下的泛在式新型图书馆服务模式}

\section{研究}

张春红，刘涛，刘玉英

沈阳航空航天大学

摘要: 随着 “互联网 + ”的概念提出, “互联网+”已经成为大趋势, 从中央到地方, 都在推动着互联网产业的迅猛发展。 图书馆如何能够把握住机遇, 利用网络环境将图书馆的服务转型, 关键是探索出“互联网+图书馆”的服务模式, 打造“互 联网+”环境下的泛在式新型图书馆, 这种模式可以不受时间、空间限制, 无时无刻满足用户需求。阐述了“互联网+”的概 念, 探讨了“互联网+” 对传统图书馆的改变, 进而提出泛在式图书馆特点及服务模式。

关键词: 互联网+; 泛在式; 新型图书馆; 服务模式

\section{1. “互联网 + ” 概念}

“互联网+”这个概念最早是由易观国际董事长兼首席执行官于扬提出, 他在 2012 年第五届移动互联 网博览会中首次提出 “互联网+” 概念, 认为 “所有传统和服务都应该被互联网改变”。而 “互联网+” 概 念的正式提出是李克强总理在第十二届全国人民代表大会第三次会议的政府工作报告中提出: “要制定 “互 联网 + , 行动计划, 推动移动互联网、云计算、大数据、物联网等与现代制造业结合, 促进电子商务、工 业互联网和互联网金融健康发展, 引导互联网企业拓展国际市场” 标志着 “互联网+”已由行业新理念、 新模式上升到国家新战略、新规划的高度 ${ }^{[1]}$ 。那么图书馆作为信息传播的重要基地, 更加离不开网络及现 代化技术, 让图书馆的资源及时快捷的推送到每一个用户身边, 让读者感受到图书馆无处不在, 随时有需 要都可以利用终端设备来利用图书馆资源, 这便是 “互联网+图书馆” 模式 ${ }^{[3,4,5,6]}$ 。

\section{2. “互联网+” 对传统图书馆的改变}

传统图书馆在互联网到来之后, 发生了翻天覆地的变化。从过去的纸质借阅, 手动签字到现在的基于 自动化系统的服务; 从茫茫书架中寻书的漫长, 到只要几分钟通过检索系统即可获得书架信息; 从没有电 
子资源服务, 只靠影印本获取国外文献信息到现在的几十个中外文数据库。沈阳航空航天大学从 2004 年 开始购买数据库, 历时 13 年, 至今已经拥有数据库 30 余个, 文献类型非常丰富, 图书、期刊、会议、报 纸、标准、博硕士论文、视频文献、课件信息等, 一站式检索, 海量信息存储, 这些都是互联网的功劳。 互联网改变了传统图书馆的服务模式, 改变了读者的阅读方式。

\section{3. 泛在式图书馆特点}

\section{1 技术是关键}

目前图书馆的现状都是年借书量, 年到馆率都在下降, 信息保障措施越来越好, 读者不愿意到图书馆, 而是更多的借助网络及终端设备获取信息。因此, 技术就显得尤为重要。泛在式图书馆发展关键要素便是 技术。

泛在式图书馆服务首要解决的问题就是技术, 高科技信息技术给图书馆的服务带来了便捷和方便, 通 信技术、网络技术、模糊识别、语音视频等技术都改变了传统图书馆的服务方式, 更多读者都是通过现代 化设备来获取信息, 因此技术就成为了泛在式图书馆的关键, 也成为了泛在式图书馆的代名词, 泛在式图 书馆是智能科技发展的结果, 是对传统图书馆发展的创新与飞跃。泛在化技术已成为人们生活中必不可少 的一部分, 泛在式图书馆正是要通过高科技技术将信息或服务等传送到人们生活中, 人们可以通过手机、 电视等科技产品随时随地地获得信息 ${ }^{[2]}$ 。

\section{2 以用户为中心}

中科院初景利教授曾提出过日心说, 既以用户为中心的图书馆服务模式。而相对于地心说的服务模式, 有如下比较:

\begin{tabular}{|l|l|l|}
\hline & 地心说（以图书馆为中心） & 日心说（以用户为中心） \\
\hline 总体规划 & 强调馆舍, 馆藏量, 经费, 人数等 & 强调对用户所提供的服务及效果 \\
\hline 服务原则 & 强调服务规则和标准 & 强调对用户所提供的服务 \\
\hline 服务对象 & 为到馆读者提供服务 & 用户在哪里服务就在哪里 \\
\hline 服务场所 & 图书馆是服务一线 & 实验室、课题组是服务一线 \\
\hline
\end{tabular}

上表中比较结果可以看出, 新型图书馆如果想要更好的发展, 就要调整传统服务模式, 完全以用户为 中心, 想用户所想, 及用户所及。将图书馆的服务重点放在如何能够满足用户的需求, 只有这样, 才能够 提高图书馆的服务能力, 服务水平。

\section{3 泛在式服务}

泛在式服务就是将图书馆的服务延伸到图书馆的围墙以外, 使得图书馆的服务无处不在, 让用户随时 随地想用图书馆资源和服务的时候, 都能够找到合适的方式来了解。图书馆服务的泛在化, 首先是服务对 象和服务范围更加广泛, 未来图书馆的服务对象不仅仅局限在本单位的读者服务范围, 而是通过网络能够 服务更多的用户, 这样才能发挥资源的最大利用率。服务范围也从图书馆的馆舍延伸到教师办公室、学生 宿舍、用户家里、读者手中。其次是服务功能和服务内容更加多样, 图书馆可以为用户提供除文献服务之 外的更多服务内容, 基于知识服务下的规划综合知识资源, 建立机构知识仓储; 为用户提供数据分析, 方 
便用户决策; 熟悉掌握文献管理工具的使用, 为用户提供推介 ${ }^{[9]}$ 。

\section{4. “互联网+”环境下的图书馆信息服务模式}

\section{1 重视 “互联网+图书馆” 顶层驱动}

互联网+环境下的图书馆发展, 一定首先要管理层改变观念, 了解先进技术。所以管理层要树立危机 感、压力和动力。管理层要有魄力, 有改变的意志。敢于 “动奶酪”, 发挥全员主观能动性。图书馆的业 务布局、部门设置、岗位设定需要结合图书馆发展进行的持续调整。结合学校引进人才计划, 不断引进和 培养领军人物。恰当的运用激励, 加强图书馆研究的能力, 发挥技术的作用, 没有研究能力就没有创新能 力。管理层是主导图书馆发展的主要因素, 只有图书馆的管理层能够及时掌握最新的技术, 图书馆的先进 理念，才能引领图书馆更快的发展。

\section{2 加强人才的全方位培养}

图书馆想提高服务能力，就一定要提高图书馆馆员的个人服务能力。将图书馆的人员能力整体提升， 随之而来的图书馆服务能力也将大大提高。打造泛在式图书馆服务模式, 就要将人员结构也做相应的转变。 无论从准入条件和学历方面, 都要做好严格的把控。

\begin{tabular}{|l|l|l|}
\hline 图书馆类型 & 传统图书馆人员结构 & 泛在式图书馆人员结构 \\
\hline 工作定位 & 文献工作者 & 知识工作者 \\
\hline 学历 & 本科及以下 & 硕士及以上 \\
\hline 准入标准 & 没有要求 & 职业资格准入 \\
\hline 人员服务能力 & 人海战术 & $\begin{array}{l}\text { 学科专长+情报能力+工具 } \\
\text { 使用能力 }\end{array}$ \\
\hline
\end{tabular}

\section{3 服务途径多样性}

利用微信微博开展图书馆各项工作。目前很多高校都已经开通微信服务号或者公众号, 读者在微信平 台上可以进行借阅、书目查询, 也可以办理解绑、绑定读者证业务, 此外还能够获取书目推荐、热门图书 等信息。大部分开通公众号的图书馆都能够查阅本馆信息, 如馆长信箱、联系我们、开馆时间、馆藏布局、 预约等。

此外有些高校图书馆老师开通微博, 梳理检索、查新等业务工作, 帮助读者更好进行数字资源利用, 深受读者喜欢。

互联网+环境下的图书馆的服务途径可谓是丰富多彩, 读者可以借助手机、IPAD 等终端, 依托网络, 安装 APP, 获取图书馆数字资源信息。目前, 已经有很多数据库开通手机服务模式, 读者只要安装 APP, 就可以在自己终端畅游信息资源。

\section{4 服务空间泛在化}

泛在式图书馆的服务就是要让读者感受到无处不在。现阶段大部分图书馆员认为自身的工作离不开图 书馆, 这与同样利用知识开展工作的医生、律师、咨询师、教师有很多不同。其实图书馆员同样可以把自 己的工作带到图书馆围墙之外。就像医生、律师、教师一样, 离开了医院、律师事务所、办公室同样也能 
有效地开展工作。可见图书馆员对工作的环境和设施过于依赖, 也说明图书馆员目前还不是真正意义上的 知识工作者, 还不能主要运用自己的专业知识和技能提供服务工作。在今天的数字化、网络化、信息化、 知识化时代, 图书馆员的核心能力必须重构, 从文献服务和信息服务, 转变为知识服务。这是今后若干年 对图书馆员能力的挑战 ${ }^{[9]}$ 。

\section{5. 结语}

在以数据化为特征和标志的深度信息化时代, 图书馆面临前所未有的机遇与挑战。图书馆不仅要与互 联网做加法, 加快向复合型图书馆转型, 还要发扬互联网精神, 借鉴互联网优势, 打造新型图书馆, 充分 利用互联网技术层面的支持扩大自身服务的半径, 寻求图书馆自身服务方式的不断创新与转化, 满足读者 的各项需求, 更好地体现图书馆以读者为本的工作理念, 使 “互联网十图书馆” 真正实现, 让图书馆无所 不在, 成为学校工作环境中不可或缺的公共空间之一 ${ }^{[7,8]}$ 。

\section{参考文献:}

[1] 鄀小燕，郭斐. “互联网+” 时代下图书馆服务的创新探索及分析．图书馆杂志 2016-11-17

[EB/OL]ht tp://www. cnki. net/kcms/detai 1/31.1108. G2. 20161117. 1437. 020. html

[2] 初丹. 论图书馆的泛在化服务_以用户为中心重构图书馆服务模式 [J]. 科技情报开发与经济. 2015, 25(21):94-95

[3] 腾讯科技. 于扬: 所有传统和服务应该被互联网改变 [EB/OL]. [2012-11-14]. http://tech. qq. com/a/20121114/000080. htm.

[4] 中国青年网.马化腾两会提案大谈 “互联网+”

[EB/0L]. [2015-3-5]. http://www. netofthings. cn/Guo Nei/2015-03/5507. htm1.

[5] 赵振. “互联网+”跨界经营: 创造性破坏视角 [J]. 中国工业经济, 2015 (10) :146.

[6] 人民网. 2015 年政府工作报告（全文实录）

[EB/OL]. [2015-3-5]. http://1ianghui. people. com. cn/2015npc/n/2015/0305/c394298-26642056. htm1.

[7] 中国经济网. 用 “互联网十” 和 “图拕馆十”成就全民说读

[EB/OL] . [2015-04-24]http://www. ce. cn/Cuiture/gd/201501/24/t20150424_5201181. shtml

[8] 沈杰.互联网_时代医院图书馆泛在化服务探讨 [J]．医学信息学杂志２015, 36(11) :64-67

[9] 初景利, 吴冬曼. 论图书馆服务的泛在化_以用户为中心重构图书馆服务模式 [J]. 图书馆建设，2008（4）：62-65

\section{References:}

[1] Yan Xiaoyan, Guo Fei. Exploration and Analysis of Library Service Innovation under "Internet plus". Library Journal 2016-11-17 [EB/OL] http://www.cnki.net /kcms/detail /31.1108. G2.20161117.1437.020.html

[2] Chu Dan. On the Universal Service of Library _ The Reconstruction of Library Service Model Based on Users [J]. Development of Science and Technology Information and Economy. 2015, 25(21):94-95

[3] Tencent Technology. Yu Yang: All Traditions and Services Should Be Changed by the Internet [EB/OL]. [2012-11-14]. http://tech.qq.com/a/20121114/000080.htm.

[4] China Youth Network. Ma Huateng talk about "Internet plus" in NPC and CPPCC [EB/OL]. [2015-3-5]. http://www.netofthings.cn/Guo Nei/2015-03/5507.html.

[5] Zhao Zhen. "Internet plus" cross-border business: Creative destruction Perspective [J]. 
Chinese Industrial Economy, 2015 (10):146.

[6] People's network. The government work report in 2015 (Record full text) [EB/OL]. [2015-3-5]. http://lianghui.people.com.cn/2015npc/n/2015/0305/c394298-26642056.html.

[7] China Economic Net. Using "Internet plus" and "Library plus" to achieve universal reading [EB/OL]. [2015-04-24] http://www.ce.cn/Cuiture/gd/201501/24/t20150424_5201181.shtml

[8] Shen Jie. Internet_ Discussion on Universal Service of Hospital Libraries in the Times [J]. Journal of Medical Informatics 2015,36(11):64-67

[9] Chu Jingli, Wu Dongman. On the Popularization of Library Service _ The Reconstruction of Library Service Model Based on Users [J]. Library Construction, 2008(4): 62-65 